\title{
Paying for Disease Management
}

\author{
PHILLIP LEVY, M.D., M.P.H., F.A.C.E.P., ${ }^{1}$ ROBERT NOCERINI, M.D., M.H.S.A., ${ }^{2}$ \\ KYLE GRAZIER, M.S., M.P.H., D.R.P.H. ${ }^{3}$
}

\begin{abstract}
Disease Management (DM) first appeared in the United States in the early 1990s. Since then its incorporation into health plans has increased dramatically, yet proof of its effectiveness in terms of quality improvement and cost reduction remains to be seen. The following review provides an exploratory analysis of the basic principles of DM, its evolution and differences from traditional managed care, the ways in which programs are currently being used in the private and public sectors, and the challenges to determining a payment structure for incorporating DM into the current health insurance system. (Disease Management 2007;10:235244)
\end{abstract}

\section{INTRODUCTION}

$\mathbf{T}$ HE ORIGINS OF DISEASE MANAGEMENT (DM) in the United States can be traced to the early 1990s, when managed care organizations (MCOs) began to recognize the impending failure of prior cost-containment efforts such as capitation and utilization review. ${ }^{1,2}$ What emerged from this "self-realization" was a system that no longer focused on influencing treatment decisions to control costs but concentrated on education and empowerment of patients to become active decision makers. ${ }^{2}$ The underlying belief was that cost reduction and health outcome improvement could be achieved through enhanced disease process understanding and concern for the psychological aspects of health. Since its inception, DM has steadily grown in popularity, with $97 \%$ of nationwide health plans pursuing some related option in their benefit offerings. ${ }^{3}$ The financial implications of this are enormous, with a tenfold increase in DM industry revenues (from $\$ 70$ million to $\$ 713$ million) between 1997 and 2003. ${ }^{2,3}$

The appeal of DM is based on its functional design, which centers on the provision of health care services through the use of coordinated, interdisciplinary clinical teams whose focus is the cost-effective optimization of health outcomes for patients with specific disease entities such as diabetes, heart failure, and chronic obstructive pulmonary disease. ${ }^{1,4}$ The general model of DM is one that employs claims data analysis to identify the most costly conditions among enrollees of a health plan with subsequent patient-level stratification on the basis of utilization patterns. ${ }^{4,5}$ At the hospital or health care system level, the compilation of patients into a disease registry is a commonly employed method that enables generation of epidemiological data and com-

\footnotetext{
${ }^{1}$ Department of Emergency Medicine, Wayne State University School of Medicine, Detroit, Michigan.

2 Doctor's Hospital of Manteca, Manteca, California.

${ }^{3}$ University of Michigan School of Public Health (Department of Health Management and Policy) and School of Medicine (Department of Psychiatry), Ann Arbor, Michigan.
} 
parison, using objective measures, with other institutions or national averages. However identified or targeted, condition-specific interventions for these patients can then be implemented with the following general goals: ${ }^{4}$

- Dissemination of (and education in) evidenced-based treatment guidelines for patients and their providers with benchmarking, performance monitoring, and provision of timely feedback

- Coordination of care through use of midlevel providers and collaborative practice models

- Objective patient assessment through administration of wellness and health surveys

- Patient education to enhance disease process understanding and promote lifestyle modification and treatment compliance

- Regular patient outreach at specified intervals using direct office or home visits or telephone evaluations

- Provision of patient "tool kits" (eg, blood pressure monitors, scales) to enable better self-care

Significant variation on this theme can exist, particularly with regard to clinical team structure, protocol or guideline development, incorporation of technology, and product delivery. The screening process for patients and group composition also can vary greatly depending on individual health plan tolerance for sensitivity and specificity. Additionally, the ac- tual provider of DM services can differ, with some organizations or institutions creating de novo internal programs and others outsourcing to vendors. ${ }^{3}$

As the concept of DM continues to evolve, new trends have begun to emerge. Common among these are a move away from a disease focus to a beneficiary focus and from high-risk individuals to at-risk populations. ${ }^{6}$ This represents an important evolution in the approach to $\mathrm{DM}$, one that values proactive rather than reactive medical care. The vision behind this shift is a medical system that redirects its efforts from the current state of decompensation avoidance for the infirmed to prevention and the prolongation of wellness for those with a predisposition to disease development. Other trends are highlighted in Table 1.

Inherent in any DM design is continuous quality assessment, with value placed on both immediate and long-term accomplishments. Measurement of quality is often focused on structural elements and process or performance indicators with benchmarking to predefined levels. ${ }^{5}$ Assessment of process improvement is particularly important to enable understanding of how DM interventions have impacted clinical management. Outcome measures such as hospital admissions or readmissions, length of stay, emergency department visits, patient-assessed quality of life, and cost provide valuable information with regard to process effectiveness and are often sought to validate the return on investment for DM. One

\begin{tabular}{|ll|}
\hline \multicolumn{1}{|c|}{ Table 1. } & Trends in Disease Management Design \\
\hline Existing & \multicolumn{1}{c}{ Emerging } \\
\hline Case based & Population based \\
Cost management & Risk management \\
Opt-in recruitment & Opt-out option \\
Medical care coordination & Self-care management and care coordination \\
Patient education & Support for behavioral change \\
Fragmented patient data & Composite patient data \\
Written guidelines & Clinical decision support systems \\
Payment tied to services & Payment tied to population outcomes \\
\hline \multicolumn{2}{c}{ SOURCE: Foote, S.M. Population-based disease management under fee-for } \\
service Medicare. Health Aff (Millwood). 2003; W3-342-56.
\end{tabular}


must exercise caution, however, when interpreting such metrics given the inherent difficulty of identifying appropriate comparator groups and the potential for confounding from environmental variables.

\section{DOES DM WORK?}

A direct answer to this question is elusive and depends in large part on the parameters used to determine success. The 2 main spheres for deliberation are cost and quality, neither of which is independent of the other.

At its roots, DM evolved as a means to address the high cost associated with underutilization and overutilization of services. Early proponents envisioned a system that would lower immediate costs through a reduction in unnecessary or redundant care and lower future costs through prevention of premature morbidity. ${ }^{3}$ Causal linkage with a positive return on investment, however, has remained elusive, in part because of a general analytic focus on process rather than costing metrics. ${ }^{5}$ Those investigations that have attempted to evaluate economic outcomes have been hindered by an absence of appropriate control patients and a general reliance on longitudinal, pre/post study design. ${ }^{5}$ Such methodological limitations increase the potential for confounding from selection bias and may overestimate DM effectiveness by failing to control for the statistical phenomenon known as regression to the mean (ie, the natural tendency for outliers to revert to the mean irrespective of intervention). 5,7 Additionally, the overall analytical framework has been inconsistent. Some studies have reported cost data primarily based on hospital resource utilization (ie, admissions, length of stay, emergency department visits), while others have included both administrative and start-up costs. 5,8

In one of the more comprehensive studies to date, Fireman et al evaluated costs associated with 4 conditions (coronary artery disease, heart failure, diabetes, and asthma) over a 7year period (1996-2002) for several thousand patients who were part of the Kaiser Permanente heath plan in Northern California. ${ }^{9}$ DM was implemented gradually with $14 \%$ of pa- tients with asthma, $20 \%$ of coronary artery disease patients, and 1 out of 4 diabetes and heart failure patients participating by 2002 . While there was a general trend toward improvement of quality indicators, such as lipid, glycemic, and blood pressure control, costs increased substantially for all 4 conditions. Though emergency department and physician clinic visits declined, pharmacy costs were noted to rise sharply. Hospital costs rose as well but by a lower percentage than their comparator group (age- and gender-matched plan members without each respective disease), leading the authors to question whether DM may have contributed to cost erosion rather than absolute reduction. Comparison to a control population of plan members with the same disease profile/severity who were not enrolled in the DM program would have added valuable information, particularly with regard to the relative degree of cost divergence from a baseline state, but this was not included in the original analysis. Thus while these data do suggest a potential for cost containment, they do not offer a definitive conclusion that DM can improve quality while lowering costs. The results of the Kaiser Permanente experience are similar to prevailing evidence on DM cost-effectiveness ${ }^{10}$; given the overall limitations of existing data, delineation of the net effect is challenging and more study on the matter is needed.

Clearly it is difficult to extrapolate from existing economic analyses of DM, and generalization of reported findings to broader patient populations must be approached with caution. The benefit of DM, however, may not be in cost saving per se but rather in the adoption of a cost-effective, value-added approach to care of the chronically ill. ${ }^{9}$ Though metrics of quality vary across studies, process measures and clinical outcomes do appear to improve uniformly with DM. ${ }^{3,10}$ Additionally, patient satisfaction with DM is high, ${ }^{3}$ a finding that clearly aligns those who provide it with the patient-centered principle of health care as put forth by the Institute of Medicine. ${ }^{11}$ Thus, it appears that DM is working to improve overall quality, which, in the end, may be more important to society than substantiation of the business case. 


\section{HEART FAILURE: A CASE IN POINT}

Heart failure (HF) provides a good example of a condition that would empirically seem to be amenable to cost and quality improvement through adoption of a DM approach, but it suffers from the existence of inconclusive evidence. HF accounts for $43 \%$ of all Medicare expenditures and $1 \%$ to $2 \%$ of total US health care costs ( $\$ 40.1$ billion in 2006). $6,12,13$ Nearly $70 \%$ of this is related to acute, inpatient treatment, which has led to the development of multidisciplinary strategies for avoidance of decompensation and improved outpatient management.

HF-specific DM programs appear to be effective from a quality standpoint, with reports of a $40 \%$ to $60 \%$ reduction in readmissions (number needed to treat $=11$ ), diminished hospital length of stay (from 6.2 to 4 days), and enhanced patient satisfaction/quality of life. ${ }^{14-25}$ Furthermore, HF-specific DM programs have been shown to improve process measures such as daily weights and dietary/medication compliance while empowering patients to self-manage their conditions. Demonstration of economic benefit, however, has been inconsistent with a handful of small hospital-based studies showing savings (ranging from $\sim \$ 1068$ per quality-adjusted life year gained in 1 study to a median decrease in annual costs of $\$ 8571$ per patient in another) and a large Kaiser Permanente analysis of $>25,000$ patient years displaying a $22 \%$ cost increase. $9,16,25,26$ These data highlight the paradox of DM, whereby seemingly dramatic cost savings from a reduction in hospital-based resource utilization may be usurped by the added spending associated with intensive outpatient care.

Such analyses, however, are particularly vulnerable to case-mix biases, with diminished margins or even increased expenditures resulting from the inclusion of both low- and high-risk patients. ${ }^{27}$ This point is more than trivial and highlights the importance of disease severity adjustment to avoid artifactual increases (or decreases) in spending due to a preponderance (or dearth) of resource-intensive program participants. Complex modeling with segregation based on utilization (eg, financial, time, facility) may be required, and the need for robust biostatistical and epidemiological expertise is clear. Despite the absence of proven cost savings, DM has been endorsed by the American College of Cardiology and the American Heart Association as a universal recommendation for institutions that provide treatment to HF patients. ${ }^{28}$

\section{INCORPORATING DM INTO EXISTING HEALTH CARE COVERAGE}

As evidenced by the data presented, thirdparty health insurers clearly believe in DM; therefore, incorporation of a DM option into coverage packages for those firms with an insurance structure based on subcontracting is relatively easy. For companies or institutions that self-insure, however, addition of a DM program may be more of a challenge. The primary obstacle is the lack of infrastructure, which must either be developed internally or outsourced. Creation of a DM program de novo offers the opportunity to tailor the design to specific needs, with the liberty of defining which disease entities to include and how to carry out the various programs. A typical methodology for DM program design is presented in Table 2. The greatest detractor from this approach would be the significant upfront costs associated with the procurement of essential components, such as office space, personnel, and information technology. Given the uncertainty of costs and benefits and the prospect for nonrecovery of such a substantial financial investment, trepidation is understandable.

An attractive alternative with less risk would be to arrange for vendor contracting. To achieve this, firms directly outsource or use a third-party insurer as an intermediary. The potential benefit of this would be a reduction in front-loaded costs and risk sharing for potential losses. A corollary to this, however, would be an abrogation of primary responsibility for the program and a decrease in autonomy of program design. Additionally, such contracted arrangements would be subject to the constraints of existing agreements between insurers and DM vendors (eg, Aetna with both Air- 
Table 2. Stepwise Methodology for Disease Management Design

1. Identification of at-risk populations of individuals through:

a. Coding groups

b. Health risk appraisals

c. Physician and patient referrals

2. Stratification of individuals by risk:

a. Assess utilization, cost

b. Clinical record

c. Physician-administered patient wellness surveys such as the Patient Health Questionnaire (PHQ9)

3. Confirmation of status:

a. Clinical record

b. Patient symptoms

c. Health risk appraisal

4. Intervention activities include, but are not limited to:

a. Case management, tele-monitoring, critical pathways, home health

b. Essentially anything that makes it easier for patients to adhere to their prescribed regimen, and anything that helps tighten the communication loop between patient and provider.

5. Measurement of outcomes, mainly objectively quantifiable data:

a. Healthcare utilization

b. Cost

c. Readmission rates

d. Length of stay

e. Emergency department visit rate

f. Office visit rate

g. Quality of life

6. Appraisal of program effectiveness:

a. Goal-oriented performance measures

i. Patient side

ii. Physician side

iii. Plan side

Source: Mayzell G. Disease management. Jacksonville Medicine. 1999;50:380-382.

Logix and Lifemasters Supported Self-Care Inc., CIGNA with American Healthways, and Humana with CorSolutions), ${ }^{6}$ creating the potential for fragmentation of care.

Beyond the direct financial implications of DM to the insuring agent, this type of programmatic shift would dramatically impact plan providers and contracted hospitals. In some cases, providers would have to cede care of their patients to the DM program, while others may lose revenue from decreased admissions or referrals. Similarly, hospitals also would stand to lose revenue, as the locus of care would shift from inpatient to office- or home-based. DM implementation, therefore, would require a restructuring of existing reimbursement arrangements. Clear direction in this regard is lacking, however, which has resulted in some level of resistance from the necessary health care stakeholders.

\section{PAYMENT FOR DM}

The absence of a basic reimbursement rate for DM efforts exists as a significant barrier to implementation and some potential reasons for this are outlined in Table 3. When considering

\begin{tabular}{l} 
TABle 3. Potential ReAsOns For the \\
Lack OF a Consistent PAyment \\
Structure FOr Disease Management \\
\hline - Diverse programs with many alternative options \\
for configuration and process \\
- A variety of third-party private payers, many of \\
whom have exclusive contracts with commercial \\
vendors \\
- Differing insurance coverage options \\
- Parallel structuring of public assistance and \\
private insurance in the United States \\
- High turnover rate in private and some public \\
health plans
\end{tabular}


potential models of payment, perhaps the most important variable is the valuation perspective on DM-directed physician activities. This is not a simple task and there are widely divergent schools of thought. Some consider DM-related health care duties to be no more than "good practice" and argue that they should not, in and of themselves, be subject to an independent reimbursement. Others counter that, though true to some degree, financial reward must be included to cover the additional time and attention to detail that results from incorporation of DM into routine practice. Many physicians, in fact, view these added tasks as uncompensated mandates, a situation that clearly must be rectified to enable the successful development of DM programs.

The decision to adopt a DM program must be seen as having long-term implications, particularly for those organizations that embark on internal program development. While current DM programs exist largely through arrangements with private insurers, expansion into Medicare and Medicaid is clearly on the horizon. Given the inevitability of this paradigm shift in the health care sector, ubiquitous DM program availability and broad consumer demand should be anticipated. A firm understanding of existing payment models will help plan administrators delineate a priori reimbursement arrangements, thereby facilitating more seamless implementation while allaying provider concerns about the potential financial impact of DM.

\section{PRIVATE INSURANCE}

Nearly all private insurers use contracted DM vendors and, in general, payments are tied to an activity, a responsibility, or an outcome. Private health plans typically pay for DM in 1 of 3 ways:

- A per member per month (PMPM) basis is the most common and resembles traditional capitation models with use of risk adjustment premiums. Bundles of services are created for specified conditions with an established price for their provision.

- A "pay for performance" (PFP)-type struc- ture, which ties reimbursement to prespecified goals such as process (eg, blood pressure reduction in $80 \%$ of members) or outcome (eg, 50\% reduction in HF admissions) achievements. Ideally, payers would like to expand this to include cost reduction, but identification of an appropriate comparison is problematic.

- Defined DM activities with an established price for each, which resembles traditional fee-for-service (FFS) models. Services provided may be more limited and are generally only selected if they have proven (evidenced-based) process or outcome benefits.

On the surface, it appears that a physician-induced moral hazard typical of traditional insurance can creep into DM programs with the above design characteristics. A physician who is paid for defined DM activities has an incentive to increase those activities and/or enroll more patients in a DM program. Similarly, a physician who is paid on a PMPM basis may withhold or stint on care. These concerns could be mitigated, however, if enrollment criteria consist of objective measurements, such as blood pressure or blood sugar levels, and the DM activities are predetermined from a set program. Hybrid designs which incorporate a PFP structure with either a PMPM or a defined DM activity arrangement may be the ideal balance.

With the evolution of a DM industry, many of the above concerns have been removed, mainly by reassigning responsibility to the subcontracting DM firm and by concentrating on predetermined outcome and performance measurements. Typically, the DM firm is subcontracted by an insurer, self-insured group, or medical practice to take responsibility for a population of patients, and payment is usually tied to quantifiable process and outcome measures. Additionally, a group practice can use these methods in-house and similarly sidestep the above improperly aligned incentives. Mechanisms for physician reimbursement in this setup are less clear. Ultimately, this may require subordination of financial considerations for the physician to the practice of enhanced patient care.

One approach that has been endorsed by the American Medical Association is the develop- 
ment of unique Current Procedural Terminology (CPT) codes for care management. Using various $\mathrm{CPT}$ codes for differing tasks, primary care physicians can then quantify the amount of activity added from DM-based care and request additional reimbursement for those services. In essence, the coding system could be used to make the case that these are patients for whom additional services are rendered and for which payment should be received. Practice groups could also use the codes for internal cost accounting and to reward individual physician performance. While potentially promising, this approach remains reliant on the willingness of private insurers to accept the code and pay accordingly.

\section{MEDICAID}

Medicaid defines DM programs in 1 of 2 ways, as a medical service or as an administrative function. ${ }^{4}$ To qualify as a medical service, it must include direct services (eg, medical assessments, disease and dietary education, instruction on health self-management, and medical monitoring) provided by a licensed provider. Such plans qualify for Federal financial participation, which is reimbursed at the state's regular Federal Medical Assistance Percentage (FMAP) rate. Several different models can meet the requirements for designation as a medical service. Common arrangements include ${ }^{4}$ :

- Through individual providers, provider groups, or Community Health Centers (CHC) that have received specialized training and can bill on a FFS basis for their DMrelated services.

- The Enhanced Primary Care Case Management Program, which uses primary care case managers to coordinate beneficiary health care for a variety of conditions. Under this design, Medicaid pays an enhanced case management fee over and above the regular FFS reimbursement schedule for other services provided.

- Contract agreements with Disease Management Organizations (DMOs). These groups manage overall care of the beneficiary with- out pre-authorization or restriction of services. Programs designed in this fashion qualify as a Prepaid Ambulatory Health Plan and thus are subject to partial managed care regulation.

Medicaid reimbursement under DMO contracted arrangements vary by state, but generally are based on 1 of the following 3 designs: ${ }^{3,4}$

1. Flat Fee (ie, PMPM for a targeted population), which includes the cost of all administrative services provided but not direct patient care services, such as office visits and provision of prescriptions. Under the Flat Fee structure, covered services include:

- Provision of education materials and protocols

- Treatment guideline consultations

- Protocol compliance monitoring (both patient and provider)

- Case management activities (eg, telephone follow-up, home visits)

- Patient database management with cost and claims analysis

- Case-mix analysis (ie, assessment of patient disease severity and acuity)

- Assessment of patient outcomes

- Patient satisfaction and wellness surveys

- Due process for issues related to beneficiary enrollment/disenrollment and grievances

- Performance reporting and continuous quality improvement measures

2. Flat Fee Plus Incentive, which includes the same PMPM payment schedule as Flat Fee plus a performance incentive based on meeting predetermined clinical and economic objectives. Minimum goals are not set and Medicaid plans that adopt this design risk a negative return on investment for the administrative fees if savings are not realized.

3. Performance Based with attached liability (termed Total Risk) for complete repayment of administrative fees if minimum cost savings and performance objectives are not achieved. Within this design, Shared Savings arrangements exist in which the DMO can claim a pre-negotiated proportion of the difference between actual Medicaid pay- 
ments on behalf of beneficiaries (including specified claims payments and MediPass case management fees) and the "baseline" payment (the estimated amount Medicaid would have paid in the absence of DM). Calculations for the Shared Savings are state-specific and can be complex; detailed methodologies for cost savings and baseline payments exist and are available on a state-by-state basis. Discrepancies between Medicaid and DMO estimates are adjudicated through annual reconciliation procedures.

DM programs that are limited to administrative activities are often established through contracted arrangements and are eligible for Federal matching funds at the standard administrative rate of $50 \% .{ }^{4}$ Such activities generally do not involve direct patient care or contact with beneficiaries themselves, but instead concentrate their efforts on provider practice patterns to improve quality. Common administrative DM services include guideline adherence monitoring, provider skills development, and utilization data management. ${ }^{4}$

Under Medicaid, DM can be implemented as a benefit by state plan amendment, which requires maintenance of beneficiary freedom of choice and statewide availability, or through submission of waivers, which permit more restrictive design and the option to mandate enrollment. ${ }^{29}$ Most of Medicaid's DM programs exist within their MCO programs, but there has been a call for development within the FFS segment. ${ }^{3}$ Though some states lack FFS-based DM programs, they do represent a more direct form of reimbursement in which billing is based on actual activity (rather than capitation). Development of CHCs and DM demonstration projects may enable program growth within the Medicaid framework, but a general dependence on Federal grant procurement for financial support is a limiting factor. Partnership with the pharmaceutical industry is a potential alternative source of funding. However, pharmaceutical funds that are directed toward DM are considered a supplemental rebate and must be shared with the Federal government at an amount based on the state's FMAP.3,4

\section{MEDICARE}

Medicare is uniquely situated to take advantage of DM programs and to be a pioneer in their development. Medicare beneficiaries are ideal candidates for DM because they have a high prevalence of chronic conditions and they are also enrolled in Medicare longer that most individuals in private plans. At present, DM programs in Medicare only exist as demonstration projects. Potential problems with incorporating DM into Medicare include the high rate of FFS participants (nearly 90\%) and the absence of accurate claims data, which is largely a consequence of the variability in prescription drug coverage. ${ }^{6}$ The general structure and payment methods of a Medicare DM program would likely be similar to the Medicaid DM programs but, at present, information is limited. Of the $4 \mathrm{DM}$ demonstration projects that have been funded by the Centers for Medicare and Medicaid Services, the majority are based on coordinated care, case management design. Only 1 capitated DM program (inclusive of risk-adjusted premiums) is being evaluated; it will combine DM support services with traditional FFS Medicare benefits. ${ }^{6}$

\section{CONCLUSIONS}

Over the past half century, the American health care system has structured itself around a payment system that rewards volume of care but not quality or outcomes of care. Managed care was the beginning of a shift in focus to paying for process and outcomes, but its incentives and restrictions were structured in ways that undermined its goals of decreasing costs and increasing quality. DM programs could be much more effective at completing this shift in payment, but if they succeed they will threaten the livelihood and existence of many individuals and institutions in our current health care system. With our current payment methods, hospitals and procedure-intensive specialties of medicine will stand to lose from this change. Decreasing patient visits, procedures, and hospitalizations decreases revenue. If the predicted quality improvements of 
DM are actually realized, and if they are significant, there is the possibility that those not in "preventive" areas of medicine will face decreased demand for their services. The outcome of this scenario could be resolved through traditional market forces: reduced demand for these services will result in a decreased supply of these services, or these individuals and institutions could find ways to develop new services that fit in the new health care environment.

The decision to adopt a DM program, therefore, has broad impact on patient and provider aspects of health care. Measuring and assigning value to outcomes and negotiating reimbursement are difficult aspects of DM program design. Understanding the basic models currently in place certainly will assist future financial structuring of DM programs, but more work is needed. These programs are not simple, and they have great potential to grow in scope and complexity, requiring experts in actuarial methods, statistical analysis, epidemiology, and operations research, to name a few, in order to construct the predictive models necessary to develop an ideal population-based DM program.

\section{REFERENCES}

1. Mayzell G. Disease management. Jacksonville Medicine. 1999;50:380-382.

2. Villagra VG. Integrating disease management into the outpatient delivery system during and after managed care. Health Aff (Millwood). Jan-Jun 2004;Suppl Web Exclusives:W4-281-283.

3. California Healthcare Foundation. Disease Management in Medicaid. Available at: <www.chcf.org/ documents / policy / DiseaseManagementInMedicaid 2004.pdf $>$. Accessed June 20, 2007.

4. National Pharmaceutical Council-Resources and Publications: What is Disease Management? Available at <www.npcnow.org/resources/issuearea/ diseasemanagement.asp $>$. Accessed July 20, 2007.

5. Congressional Budget Office. An Analysis of the Literature on Disease Management Programs. Available at: <www.cbo.gov/showdoc.cfm?index $=5909 \&$ sequence $=0>$. Accessed June 20, 2007.

6. Foote SM. Population-based disease management under fee-for-service Medicare. Health Aff (Millwood). Jul-Dec 2003;Suppl Web Exclusives:W3-342-356.

7. Wilson TW. Evaluating ROI in state disease management programs. State Coverage Initiat Issue Brief. 2003;4:1-6.
8. Villagra V. Strategies to control costs and quality: a focus on outcomes research for disease management. Med Care. 2004;42(4 suppl):III24-III30.

9. Fireman B, Bartlett J, Selby J. Can disease management reduce health care costs by improving quality? Health Aff (Millwood). 2004;23:63-75.

10. Ofman JJ, Badamgarav E, Henning JM, et al. Does disease management improve clinical and economic outcomes in patients with chronic diseases? A systematic review. Am J Med. 2004;117:182-192.

11. Berwick DM. A user's manual for the IOM's "Quality Chasm" report. Health Aff (Millwood). 2002; 21:80-90.

12. Stewart $\mathrm{S}$. Financial aspects of heart failure programs of care. Eur J Heart Fail. 2005;7:423-428.

13. Thom T, Haase N, Rosamond W, et al. Heart disease and stroke statistics, 2006 update: a report from the American Heart Association Statistics Committee and Stroke Statistics Subcommittee. Circulation. 2006;113: e85-e151.

14. Cline CM, Israelsson BY, Willenheimer RB, Broms K, Erhardt LR. Cost effective management programme for heart failure reduces hospitalisation. Heart. 1998;80:442-446.

15. Gwadry-Sridhar FH, Flintoft V, Lee DS, Lee H, Guyatt GH. A systematic review and meta-analysis of studies comparing readmission rates and mortality rates in patients with heart failure. Arch Intern Med. 2004;164:2315-2320.

16. Krumholz HM, Amatruda J, Smith GL, et al. Randomized trial of an education and support intervention to prevent readmission of patients with heart failure. I Am Coll Cardiol. 2002;39:83-89.

17. McAlister FA, Lawson FM, Teo KK, Armstrong PW. A systematic review of randomized trials of disease management programs in heart failure. $\underline{A m ~ I ~ M e d . ~}$ 2001;110:378-384.

18. McAlister FA, Stewart S, Ferrua S, McMurray JJ. Multidisciplinary strategies for the management of heart failure patients at high risk for admission: a systematic review of randomized trials. I Am Coll Cardiol. 2004;44:810-819.

19. Philbin EF. Comprehensive multidisciplinary programs for the management of patients with congestive heart failure. L Gen Intern Med. 1999;14:130-135.

20. Rich MW, Beckham V, Wittenberg C, Leven CL, Freedland KE, Carney RM. A multidisciplinary intervention to prevent the readmission of elderly patients with congestive heart failure. $N$ Engl J Med. 1995; 333:1190-1195.

21. Riegel B, Carlson B, Kopp Z, LePetri B, Glaser D, Unger A. Effect of a standardized nurse case-management telephone intervention on resource use in patients with chronic heart failure. Arch Intern Med. 2002;162:705-712.

22. Shah NB, Der E, Ruggerio C, Heidenreich PA, Massie BM. Prevention of hospitalizations for heart failure with an interactive home monitoring program. $\underline{A m}$ Heart I. 1998;135:373-378. 
23. Stewart S, Marley JE, Horowitz JD. Effects of a multidisciplinary, home-based intervention on unplanned readmissions and survival among patients with chronic congestive heart failure: a randomised controlled study. Lancet. 1999;354:1077-1083.

24. Stromberg A, Martensson J, Fridlund B, Levin LA, Karlsson JE, Dahlstrom U. Nurse-led heart failure clinics improve survival and self-care behaviour in patients with heart failure: results from a prospective, randomised trial. Eur Heart J. 2003;24:1014-1023.

25. Whellan DJ, Gaulden L, Gattis WA, et al. The benefit of implementing a heart failure disease management program. Arch Intern Med. 2001;161:2223-2228.

26. Capomolla S, Febo O, Ceresa M, et al. Cost/utility ratio in chronic heart failure: comparison between heart failure management program delivered by day-hospital and usual care. LAm Coll Cardiol. 2002;40: 1259-1266.

27. Riegel B, Carlson B, Glaser D, Hoagland P. Which patients with heart failure respond best to multidisci- plinary disease management? L Card Fail. 2000;6: 290-299.

28. Krumholz HM, Baker DW, Ashton CM, et al. Evaluating quality of care for patients with heart failure. Circulation. 2000;101:E122-E140.

29. Centers for Medicare and Medicaid Services. Medicaid. Available at: <www.cms.hhs.gov/home/medicaid. asp $>$. Accessed June 20, 2007.

Address reprint requests to: Phillip Levy, MD, MPH, FACEP

Assistant Professor Wayne State University Department of Emergency Medicine 4201 St. Antoine

Detroit, MI 48201

E-mail: phillevy_2000@yahoo.com 